\title{
Spectroscopy, gluon structure and polarisability of kaons in the new QCD facility at the M2 beam line at CERN SPS
}

\author{
Barbara Badelek ${ }^{* \dagger}$ \\ Faculty of Physics, University of Warsaw \\ E-mail: badelekefuw.edu.pl
}

The COMPASS++ /AMBER (proto-) Collaboration proposes a broad experimental programme for the "New QCD facility at the M2 beam line of the CERN SP", to commense after 2021. In this article, a few of unique second-generation experiments that rely on high-energy high intensity RF-separated kaon beams planned to operate after 2026 are highlighted: the kaon spectroscopy with kaon beam, a study of the gluon distribution in the kaon via prompt-photon production and a kaon polarisability measurement.

XXVII International Workshop on Deep-Inelastic Scattering and Related Subjects - DIS2019 8-12 April, 2019

Torino, Italy

* Speaker.

† On behalf of COMPASS++/AMBER (proto-) Collaboration. 


\section{Introduction}

The COMPASS experiment [1] expected to have the last year of data taking in 2021 has been very successful in studies of nucleon and other hadrons' structure and spectroscopy. It has taken data since 2002, with different beams and targets, see Table 1.

Table 1: COMPASS data taking until now: dark red colour denotes muon DIS runs with polarised deuterium, while the red with polarised hydrogen.

\begin{tabular}{|l|l|}
\hline \hline $2002-2004$ & nucleon structure $\mu-\mathrm{d}, 160 \mathrm{GeV}, \mathrm{L}$ and T polarised target \\
2005 & CERN accelerator shutdown, increase of target acceptance \\
2006 & nucleon structure $\mu-\mathrm{d}, 160 \mathrm{GeV}, \mathrm{L}$ polarised target \\
2007 & nucleon structure $\mu-\mathrm{p}, 160 \mathrm{GeV}, \mathrm{L}$ and T polarised target \\
$2008-2009$ & hadron spectroscopy; Primakoff reaction \\
2010 & nucleon structure $\mu-\mathrm{p}, 160 \mathrm{GeV}, \mathrm{T}$ polarised target \\
2011 & nucleon structure $\mu-\mathrm{p}, 200 \mathrm{GeV}, \mathrm{L}$ polarised target \\
2012 & Primakoff reaction $\left(\pi^{-}-\mathrm{C} / \mathrm{Ni} / \mathrm{W}\right), 190 \mathrm{GeV} ;$ \\
& DVCS/SIDIS $\mu-\mathrm{p}, 160 \mathrm{GeV}$, unpolarised target (test) \\
2013 & CERN accelerator shutdown, $\mathrm{LS} 1$ \\
2014 & Drell-Yan $\pi-\mathrm{p}$ reaction with T polarised target (test) \\
2015 & Drell-Yan $\pi-\mathrm{p}$ reaction with T polarised target \\
$2016-2017$ & DVCS/SIDIS $\mu-\mathrm{p}, 160 \mathrm{GeV}$, unpolarised target \\
2018 & Drell-Yan $\pi-\mathrm{p}$ reaction with T polarised target \\
$2019-2020$ & CERN accelerator shutdown, LS2 \\
\hline 2021 & nucleon structure $\mu-\mathrm{d}, 160 \mathrm{GeV}$, T polarised target \\
& (SPSC approved addendum, see A. Martin's contribution to these proceedings) \\
\hline \hline
\end{tabular}

Planning of the future of the COMPASS facility began in March 2016 with the "COMPASS beyond 2020 Workshop" at CERN, https://indico.cern.ch/event/502879/, intertwined with the CERN "Physics Beyond Colliders" initiative, https://indico.cern.ch/category/7885/. Recently a Letter of Intent by the COMPASS++/AMBER (proto-) Collaboration was published [2]. It contains a description of a broad experimental programme for the new QCD facility at the M2 beam line of the CERN SPS, planned for years after the ongoing Long Shutdown 2. A list of new projects, their main features and required hardware additions are given in Table 2. For every proposed experiment, individual instrumentation using modern detector architecture will be constructed and installed in the experimental hall EHN2, where the upgraded multi-purpose two-stage magnetic spectrometer will serve as experimental backbone of the new facility.

In Table 2 items between thick red lines, all for RF-separated hadron beams, will be highlighted in this article. The RF-separated hadron beams are foreseen to be operational after the Long Shutdown 3, planned for 2025-2026. For a discussion of other 'after-the-2021' subjects see Ref.[3]. In particular the programme for 2022-2024 ('Phase - 1') is covered at length in the proposal, Ref. [4]. 


\section{Selected items in the 'after-the-2021' programme}

In this section certain experiments planned by the COMPASS++/AMBER (proto-) Collaboration are highlighted, see Table 2. Those experiments assume a usage of the Radio-Frequency separated kaon beam, planned to be available after the LS3, i.e. after 2026.

Table 2: Requirements for future programmes at the M2 beam line after 2021 [2]. Standard muon beams are in blue, standard hadron beams in green, and RF-separated hadron beams in red, in the lower part of the table. Items between thick red lines will be discussed in this article.

\begin{tabular}{|c|c|c|c|c|c|c|c|c|}
\hline Program & $\begin{array}{l}\text { Physics } \\
\text { Goals }\end{array}$ & $\begin{array}{l}\text { Beam } \\
\text { Energy } \\
{[\mathrm{GeV}]}\end{array}$ & $\begin{array}{c}\text { Beam } \\
\text { Intensity } \\
{\left[\mathrm{s}^{-1}\right]}\end{array}$ & $\begin{array}{c}\text { Trigger } \\
\text { Rate } \\
{[\mathrm{kHz}]}\end{array}$ & $\begin{array}{l}\text { Beam } \\
\text { Type }\end{array}$ & Target & $\begin{array}{l}\text { Earliest } \\
\text { start time, } \\
\text { duration }\end{array}$ & $\begin{array}{l}\text { Hardware } \\
\text { Additions }\end{array}$ \\
\hline $\begin{array}{c}\mu p \\
\text { elastic } \\
\text { scattering }\end{array}$ & $\begin{array}{c}\text { Precision } \\
\text { proton-radius } \\
\text { measurement }\end{array}$ & 100 & $4 \cdot 10^{6}$ & 100 & $\mu^{ \pm}$ & $\begin{array}{l}\text { high-pr. } \\
\text { H2 }\end{array}$ & $\begin{array}{c}2022 \\
1 \text { year }\end{array}$ & $\begin{array}{l}\text { active TPC } \\
\text { SciFi trigger } \\
\text { silicon veto }\end{array}$ \\
\hline $\begin{array}{c}\text { Hard } \\
\text { exclusive } \\
\text { reactions }\end{array}$ & GPD $E$ & 160 & $2 \cdot 10^{7}$ & 10 & $\mu^{ \pm}$ & $\mathrm{NH}_{3}^{\uparrow}$ & $\begin{array}{c}2022 \\
2 \text { years }\end{array}$ & $\begin{array}{c}\text { recoil silicon, } \\
\text { modified polarised } \\
\text { target magnet }\end{array}$ \\
\hline $\begin{array}{l}\text { Input for Dark } \\
\text { Matter Search }\end{array}$ & $\begin{array}{l}\bar{p} \text { production } \\
\text { cross-section }\end{array}$ & $20-280$ & $5 \cdot 10^{5}$ & 25 & $p$ & $\begin{array}{l}\text { LH2, } \\
\text { liquid helium }\end{array}$ & $\begin{array}{c}2022 \\
1 \text { month }\end{array}$ & $\begin{array}{l}\mathrm{LHe} \\
\text { target }\end{array}$ \\
\hline $\begin{array}{c}\bar{p} \text {-induced } \\
\text { spectroscopy }\end{array}$ & $\begin{array}{l}\text { Heavy quark } \\
\text { exotics }\end{array}$ & 12,20 & $5 \cdot 10^{7}$ & 25 & $\bar{p}$ & LH2 & $\begin{array}{c}2022 \\
2 \text { years }\end{array}$ & $\begin{array}{l}\text { target spectr.: } \\
\text { tracking, } \\
\text { calorimetry }\end{array}$ \\
\hline Drell-Yan & Pion PDFs & 190 & $7 \cdot 10^{7}$ & 25 & $\pi^{ \pm}$ & $\mathrm{C} / \mathrm{W}$ & $\begin{array}{c}2022 \\
1-2 \text { years }\end{array}$ & \\
\hline $\begin{array}{l}\text { Drell-Yan } \\
\quad(\mathrm{RF})\end{array}$ & $\begin{array}{c}\text { Kaon PDFs \& } \\
\text { nucleon TMDs }\end{array}$ & $\sim 100$ & $10^{8}$ & $25-50$ & $K^{ \pm}, \bar{p}$ & $\begin{array}{l}\mathrm{NH}_{3}^{\uparrow}, \\
\mathrm{C} / \mathrm{W}\end{array}$ & $\begin{array}{c}2026 \\
2-3 \text { years }\end{array}$ & $\begin{array}{c}\text { "active absorber", } \\
\text { vertex det. }\end{array}$ \\
\hline $\begin{array}{l}\text { Primakoff } \\
\quad(\mathrm{RF})\end{array}$ & $\begin{array}{l}\text { Kaon polarisa- } \\
\text { bility \& pion } \\
\text { life time }\end{array}$ & $\sim 100$ & $5 \cdot 10^{6}$ & $>10$ & $K^{-}$ & $\mathrm{Ni}$ & $\begin{array}{c}\text { non-exclusive } \\
2026 \\
1 \text { year }\end{array}$ & \\
\hline $\begin{array}{c}\text { Prompt } \\
\text { Photons } \\
\text { (RF) }\end{array}$ & $\begin{array}{l}\text { Meson gluon } \\
\text { PDFs }\end{array}$ & $\geq 100$ & $5 \cdot 10^{6}$ & $10-100$ & $\begin{array}{l}K^{ \pm} \\
\pi^{ \pm}\end{array}$ & $\begin{array}{l}\mathrm{LH} 2, \\
\mathrm{Ni}\end{array}$ & $\begin{array}{c}\text { non-exclusive } \\
2026 \\
1-2 \text { years }\end{array}$ & hodoscope \\
\hline $\begin{array}{c}K \text {-induced } \\
\text { spectroscopy } \\
(\mathrm{RF})\end{array}$ & $\begin{array}{l}\text { High-precision } \\
\text { strange-meson } \\
\text { spectrum }\end{array}$ & $50-100$ & $5 \cdot 10^{6}$ & 25 & $K^{-}$ & LH2 & $\begin{array}{c}2026 \\
1 \text { year }\end{array}$ & $\begin{array}{c}\text { recoil TOF } \\
\text { forward PID }\end{array}$ \\
\hline $\begin{array}{l}\text { Vector mesons } \\
\text { (RF) }\end{array}$ & $\begin{array}{c}\text { Spin Density } \\
\text { Matrix } \\
\text { Elements }\end{array}$ & $50-100$ & $5 \cdot 10^{6}$ & $10-100$ & $K^{ \pm}, \pi^{ \pm}$ & $\begin{array}{l}\text { from } \mathrm{H} \\
\text { to } \mathrm{Pb}\end{array}$ & $\begin{array}{c}2026 \\
1 \text { year }\end{array}$ & \\
\hline
\end{tabular}

\subsection{RF-separated hadron beams}

A newly designed and constructed Radio-Frequency separated hadron beams are the main challenge of the long term future programme of COMPASS++/AMBER. The idea is based on the old Panofsky-Montague-Schnell proposals, with two RF cavities [5]. Very preliminary estimates give probable values of $80 \mathrm{GeV}$ and $110 \mathrm{GeV}$ momenta and intensity gains about 80 and 50 for kaon and antiproton beams, respectively. The latter should be compared with a $2.5 \%$ and $0.5 \%$ content of kaons and antiprotons in a standard negative hadron beam. 


\subsection{Kaon spectroscopy with kaon beam}

The goal here is to map out the complete spectrum of excited kaons, Fig. 1, with unprecedented precision using novel analysis methods. A more precise knowledge of the kaon spectrum will have a broad impact not only on low-energy QCD, but also on many processes in modern hadron and particle physics where excited kaons appear, e.g. in the study of $\mathrm{CP}$ violation in heavy-meson decays, which is ongoing at $\mathrm{LHCb}$ and Belle II.

The high-intensity RF-separated kaon beam of the upgraded M2 beam line is an univalled place to accumulate a large data set for kaon spectroscopy as most of the planned or proposed measurements in the strange-meson sector elsewhere can either not compete with the measurement proposed here or are complementary to it. Taking data over one year with a beam energy of at least 50 $\mathrm{GeV}$, about $20 \times 10^{6}$ events can be collected, which allows us to keep systematic effects under control. The main experimental challenges are high-precision vertex reconstruction, photon detection with electromagnetic calorimeters

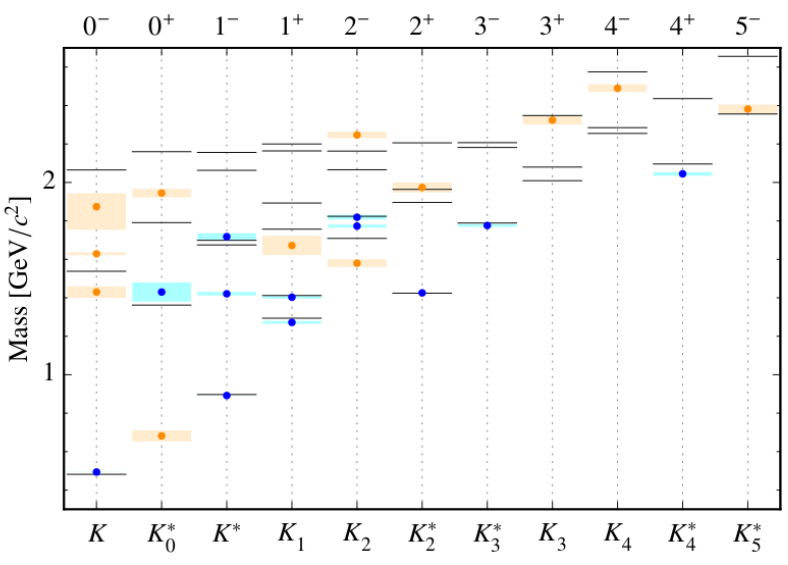

Figure 1: Excitation spectrum of strange mesons from PDG [6]. Lines mark relativistic QM predictions, blue - states listed in the PDG summary table, orange - remaining states. The states are grouped by their $J^{P}$ quantum numbers. Figure from [2].

for reconstructing neutral hadrons, and final-state particle identification, i.e. kaons have to be distinguished from pions with high efficiency over a broad kinematic range.

\subsection{Study of the gluon distribution in the kaon via prompt-photon production}

The purpose of this experiment is the study of the gluon content of charged kaons. This is of fundamental importance for understanding the internal structure of light mesons and may also shed light onto the mechanism that generates their mass. In contrast to the well-known gluon distribution of the nucleon, our knowledge on the gluon distribution in light mesons is rather limited. It can be considerably improved by studying prompt-photon production in hadronic collisions using a highenergy meson beam. Prompt photons are photons that are produced by hard scattering of partons. As kaons have heavier valence quarks, less glue is expected in them than in pions and nucleons: $\sim 5 \%(\mathrm{~K})$ vs $\sim 30 \%(\pi)$ vs $\sim 50 \%(\mathrm{~N})$.

The RF-separated kaon beam of high intensity, $\sim 5 \times 10^{6} \mathrm{~K}^{+} / \mathrm{s}$, of the upgraded M2 beam line is an unrivalled site to investigate the gluon content of the kaon. Using a beam of positive kaons with an energy of at least $100 \mathrm{GeV}$ over 1-2 years, the gluon PDF in the charged kaon will be measured using the dominant hard gluon Compton scattering process, $\mathrm{qg} \rightarrow \gamma \mathrm{q}$, in the range of $x_{g}$ above 0.05 and for $Q^{2} \sim p_{T}^{2}>7(\mathrm{GeV} / c)^{2}$. A resulting sample of the gluon Compton events should 
amount to about a million events. A separate data set will have to be taken with a beam of negative kaons in order to allow for a separation of the subdominant quark-antiquark annihilation process. Kinematic distributions and energy dependence of the prompt $\gamma$ production cross section are shown in Fig. 2. For systematic studies and also to improve the knowledge on the gluon structure of the pion, it is foreseen to also collect data with incoming pions. This can be done either in parallel or separately in a preceding running period. The basic requirements for the proposed measurement are a sufficiently high transparency of the experimental setup for the produced photons, a wide kinematic range of photon detection by the system of electromagnetic calorimeters, efficient beam hadron identification, and a dedicated calorimeter-based trigger on high- $p_{T}$ photons. We are not aware of plans to study the gluon structure of charged kaons at other laboratories.
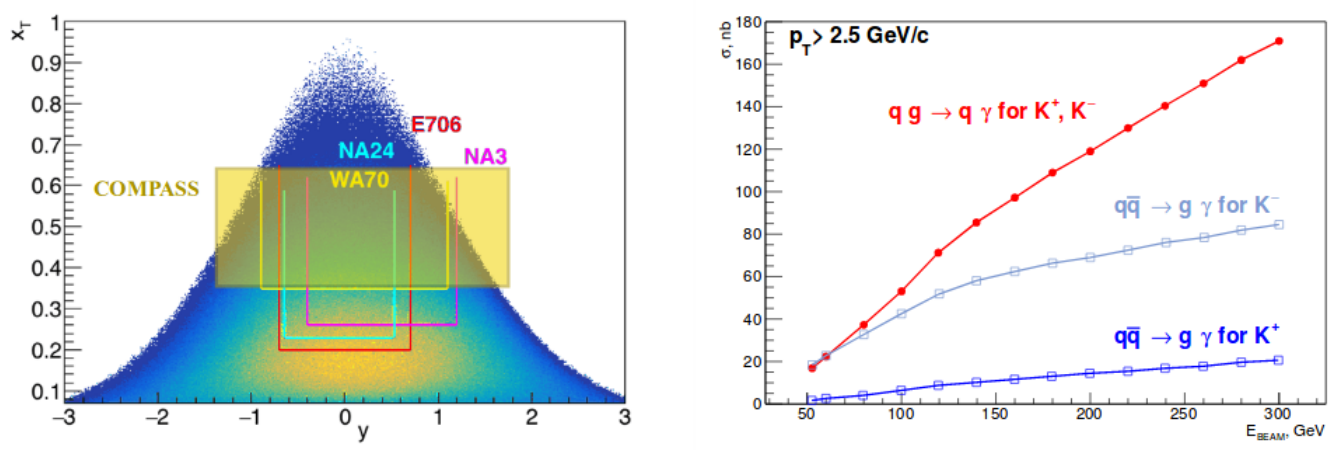

Figure 2: Left: Kinematic distribution for $x_{T}=2 p_{T} / \sqrt{s}$ vs.centre-of-mass rapidity, $y$ for the gluon Compton scattering process, for a $100 \mathrm{GeV} \mathrm{K}{ }^{+}$beam scattered off a proton target. The kinematic ranges covered by previous low-energy pion beam experiments and the possible coverage by COMPASS are also shown. Right: energy dependence of the prompt-photon production cross section for $p_{T}>2.5 \mathrm{GeV} / c$, for two production mechanisms (gluon Compton scattering, $\mathrm{qg} \rightarrow \gamma_{\mathrm{q}}$ and the background quark annihilation, $\mathrm{q} \overline{\mathrm{q}} \rightarrow \gamma \mathrm{g}$ ) and positive as well as negative kaon beams under the assumption of identical gluon distributions in $\pi$ and K. Figure from [2].

\subsection{Kaon polarisability}

The main objective of this experiment is the first measurement of the kaon polarisability. Electric and magnetic polarisabilities, which describe the rigidity against deformation by an external electromagnetic field, constitute fundamental quantities of low-energy QCD. Apart from a quite vague experimental upper limit, presently only model predictions exist for the electric polarisability of the kaon, e.g. by chiral perturbation theory $(\chi P T)$ or the quark-confinement model; they differ by a factor of four. The kaon electric polarisability can be determined employing the so-called Primakoff reaction, $K^{-} Z \rightarrow K^{-} \gamma Z$, using a high-energy high-intensity kaon beam, on a nuclear target $Z$ with $Z$ protons. Such an experimental approach allows the parallel determination of another fundamental quantity of low-energy QCD, i.e. the lifetime of the neutral pion, which is related to the chiral-anomaly hypothesis.

The RF-separated beam of the upgraded M2 beam line is a unique place for these measurements. One year of data taking with a $100 \mathrm{GeV}$ beam delivering $5 \times 10^{6}$ kaons per second will allow to determine the electric kaon polarisability with a statistical accuracy of $0.03 \times 10^{-4} \mathrm{fm}^{3}$, with the total systematic uncertainty estimated to be smaller. This accuracy is about 20 times smaller than 
that of the prediction of $\chi P T$ in the one-loop approximation and will clearly allow us to distinguish between models for the kaon polarisability. The neutral pion lifetime will be measured in parallel using those beam pions that remain in the RF-separated kaon beam. Here, the resulting statistical accuracy of about $1 \%$ will be smaller by more than a factor of two compared to the accuracy of the theoretical prediction and that of existing measurements. The proposed measurement requires advanced beam particle identification, excellent tracking capabilities at small angles and precise electromagnetic calorimeters and have presently no competitors in the world.
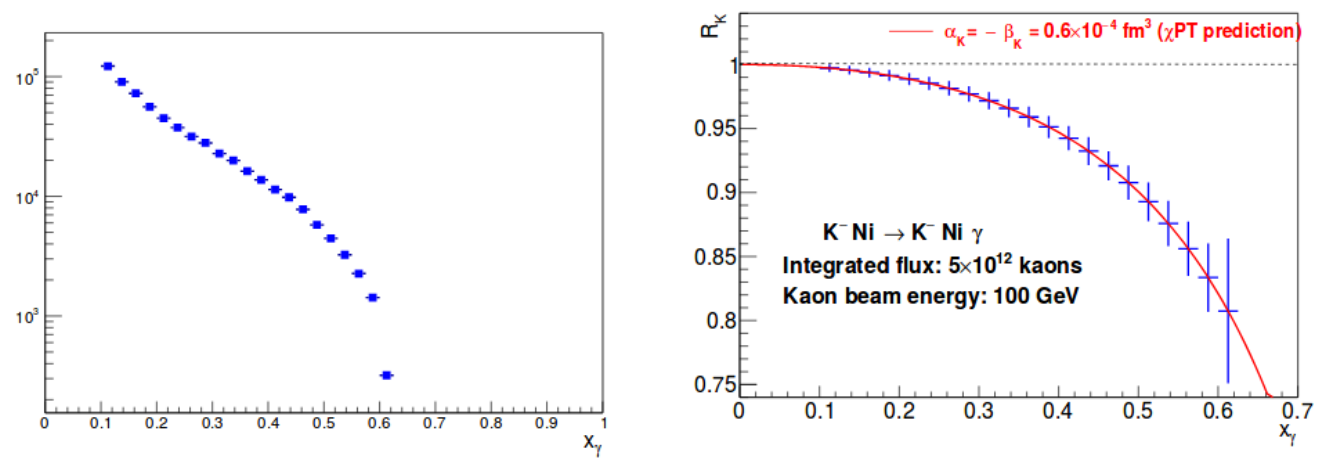

Figure 3: Left: the expected $x_{\gamma}$ spectrum for $K^{-} \gamma$ events. Right: the statistical accuracy for the measurement of the ratio $R_{K}$ of the differential cross section for the real kaon over the expected cross section for a hypothetical point-like kaon as a function of $x_{\gamma}$. The ratio $R_{K}$ corresponding to the $\chi \mathrm{PT}$ prediction is shown as a red continuous line. Figure from [2].

\section{Outlook}

A broad experimental programme for the "New QCD facility at the M2 beam line of the CERN SPS" is proposed. This unrivalled installation will provide the site for a great variety of measurements to address fundamental issues of Quantum Chromodynamics. In this article, we briefly describe a few of unique second-generation experiments that rely on high-energy highintensity RF-separated hadron beams, which could be made possible by a major upgrade of the M2 beam line. Such an upgrade, which is presently under study at CERN would make the CERN SPS M2 beam line absolutely unique in the world for many years to come. New groups are welcome to join this fascinating programe!

\section{References}

[1] COMPASS Collaboration, P. Abbon et al., Nucl. Instr. Meth. A 577 (2007) 455; ibid. 779 (2015) 69.

[2] COMPASS++ /AMBER (proto-) Collaboration, B. Adams et al., arXiv:1808.00848v6.

[3] COMPASS++ /AMBER (proto-) Collaboration, C. Dreisbach, these proceedings; V. Andrieux, ibid..

[4] COMPASS++/AMBER (proto-) Collaboration, B. Adams et al., CERN-SPSC-2019-022/SPSC-P-36.

[5] P. Bernard et al., CERN 68-29.

[6] C. Patrignani et al. (Particle Data Group), Chinese Physics C 40 (2016) 100001. 